\title{
LIX. On convection currents in a horizontal layer of fluid, when the higher temperature is on the under side
}

\section{Lord Rayleigh O.M. F.R.S.}

To cite this article: Lord Rayleigh O.M. F.R.S. (1916) LIX. On convection currents in a horizontal layer of fluid, when the higher temperature is on the under side, Philosophical Magazine Series 6, 32:192, 529-546, DOI: 10.1080/14786441608635602

To link to this article: http://dx.doi.org/10.1080/14786441608635602

曲 Published online: 08 Apr 2009.

Submit your article to this journal $[\pi$

Џll Article views: 346

Q View related articles $\llbracket$

7 Citing articles: 757 View citing articles 
LONDON, LDINBURGH, AND DUBLIN

\title{
PHILOSOPHICAL MAGAZINE
}

\author{
AND \\ JOURNAL OF SCIENCE.
}

[SIXTH SERIES ]

$D E C E M B E R 1916$.

LIX. On Convection Currents in a Horizontal Layer of Fluid, when the Higher Temperature is on the Under Side. By Lord Rayleigh, O.M., F.R.S.*

$7 \mathrm{HE}$ present is an attempt to examine how far the interesting results obtained by Bénard $\dagger$ in his careful and skilful experiments can be explained theoretically. Bénard worked with very thin layers, only about $1 \mathrm{~mm}$. deep, standing on a levelled metallic plate which was maintained at a uniform temperature. The upper surface was usually free, and being in contact with the air was at a lower temperature. Various liquids were employed-some, indeed, which would be solids under ordinary conditions.

The layer rapidly resolves itself into a number of cells, the motion being an ascension in the middle of a cell and a descension at the common boundary between a cell and its neighbours. Two phases are distinguished, of unequal duration, the first being relatively very short. The limit of the first phase is described as the "semi-regular cellular regime"; in this state all the cells have already acquired surfaces nearly identical, their forms being nearly regular convex polygons of, in general, 4 to 7 sides. The bounduries

* Communicated by the Author.

† Revue générale des Sciences, vol. xii. pp. 1261, 1309 (1900); Ann.d. Chimie et de Physique, t. xxiii. p. 62 (1901). M. Bénard does not appear to be acquainted with James Thomson's paper "On a Changing Tesselated Structure in certain Liquids" (Proc. Glasgow Phil. Soc. 1881-2), where a like structure is described in much thicker layers of soapy water cooling from the surface.

Fhil. Mag. S. 6. Vol. 32. No. 192. Dec. 1916. 20 
are vertical, and the circulation in each cell approximates to that already indicated. This phase is brief ( 1 or 2 seconds) for the less viscous liquids (alcohol, benzine, \&c.) at ordinary temperatures. Even for paraffin or spermacetti, melted at $100^{\circ} \mathrm{C} ., 10$ seconds suffice ; but in the case of very viscous liquids (oils, \&c.), if the flux of heat is small, the deformations are extremely slow and the first phase may last several minutes or more.

The second phase has for its limit a permanent regime of regular hexagons. During this period the cells become equal and regular and allign themselves. It is extremely protracted, if the limit is regarded as the complete attainment of regular hexagons. And, indeed, such perfection is barely attainable even with the most careful arrangements. The tendency, however, seems sufficiently established.

The theoretical consideration of the problem here arising is of interest for more than one reason. In general, when a system falls away from unstable equilibrium it may do so in several principal modes, in each of which the departure at time $t$ is proportional to the small displacement or velocity supposed to be present initially, and to an exponential factor $e^{q t}$, where $q$ is positive. If the initial disturbances are small enough, that mode (or modes) of falling away will become predominant for which $q$ is a maximum. The simplest example for which the number of degrees of freedom is infinite is presented by a cylindrical rod of elastic material under a longitudinal compression sufficient to overbalance its stiffness. But perhaps the most interesting hitherto treated is that of a cylinder of fluid disintegrating under the operation of capillary force as in the beautiful experiments of Savart and Flateau upon jets. In this case the surface remains one of revolution about the original axis, but it becomes varicose, and the question is to compare the effects of different wave-lengths of varicosity, for upon this depends the number of detached masses into which the column is eventually resolved. It was proved by Plateau that there is no instability if the wave-length be less than the circumference of the column. For all wave-lengths greater than this there is instability, and the corresponding modes of disintegration may establish themselves if the initial disturbances are suitable. But if the general disturbance is very small, those components only will have opportunity to develop themselves for which the wavelength lies near to that of maximum instability.

It has been shown * that the wave-length of maximum

* Proc. Lond. Math. Soc. vol. x. p. 4 (1879); Scientific Papers, vol.i. p. 361. Also 'Theory of Sound,' 2 nd ed. $\$ \$ 35 \%$, \&c. 
instability is 4.508 times the diameter of the jet, exceeding the wave-length at which instability first enters in the ratio of about $3: 2$. Accordingly this is the sort of disintegration to be expected when the jet is shielded as far as possible from external disturbance.

It will be observed that there is nothing in this theory which could fix the phase of the predominant disturbance, or the particular particles of the fluid which will ultimately form the centres of the detached drops. There remains a certain indeterminateness, and this is connected with the circumstance that absolnte regularity is not to be expected. In addition to the wave-length of maximum instability wo must include all those which lie sufficiently near to it, and the superposition of the corresponding modes will allow of a slow variation of phase as we pass along the column. The phase in any particular region depends upon the initial circumstances in and near that region, and these are supposed to be matters of chance*. The superposition of infinite trains of waves whose wave-lengths cluster round a given value raises the same questions as we are concerned with in considering the character of approximately homogeneous light.

In the present problem the case is much more complicated, unless we arbitrarily limit it to two dimensions. The cells of Bénard are then reduced to infinitely long strips, and when there is instability we may ask for what wavelength (width of strip) the instability is greatest. The answer can be given under certain restrictions, and the manner in which equilibrium breaks down is then approximately determined. So long as the two-dimensional character is retained, there seems to be no reason to expect the wave-length to alter afterwards. But even if we assume a natural disposition to a two-dimensional motion, the direction of the length of the cells as well as the phase could only be determined by initial circumstances, and conld not be expected to be uniform over the whole of the infinite plane.

According to the observations of Bénard, something of this sort actually occurs when the layer of liquid has a general motion in its own plane at the moment when instability commences, the length of the cellular strips being parallel to the general velocity. But a little later, when the general motion has decayed, division-lines runaing in the perpendicular direction present themselves.

* When a jet of liquid is acted on by an external vibrator, the resolution into drops may be regularized in a much bigher degree. 
In general, it is easy to recognize that the question is much more complex. By Fourier's theorem the motion in its earlier stages may be analysed into components, each of which corresponds to rectangular cells whose sides are parallel to fixed axes arbitrarily chosen. The solution for maximum instability yields one relation between the sides of the rectangle, but no indication of their ratio. It covers the two-dimensional case of infinitely long rectangles already referred to, and the contrasted case of squares for which the length of the side is thus determined. I do not see that any plausible hypotliesis as to the origin of the initial disturbances leads us to expect one particular ratio of sides in preference to another.

On a more general view it appears that the function expressing the disturbance which develops most rapidly may be assimilated to that which represents the free vibration of an infinite stretched membrane vibrating with given frequency.

The calculations which follow are based upon equations given by Boussinesq, who has applied them to one or two particular problems. The special limitation which characterizes them is the neglect of variations of density, except in so far as they modify the action of gravity. Of course, such neglect can be justified only under certain conditions, which Boussinesq has discussed. They are not so restrictive as to exclude the approximate treatment of many problems of interest.

When the fluid is inviscid and the higher temperature is below, all modes of disturbance are instable, even when we include the conduction of heat during the disturbance. But there is one class of disturbances for which the instability is a maximum.

When viscosity is included as well as conduction, the problem is more complicated, and we have to consider boundary conditions. Those have been chosen which are simplest from the mathematical point of view, and they deviate from those obtaining in Bénard's experiments, where, indeed, the conditions are different at the two boundaries. It appears, a little unexpectedly, that the equilibrium may be thoroughly stable (with higher temperature below), if the coefficients of conductivity and viscosity are not too small. As the temperature gradient increases, instability enters, and at first only for a particular kind of disturbance.

The second phase of Bénard, where a tendency reveals itself for a slow transformation into regular hexagons, is not 
touched. It would seem to demand the inclusion of the squares of quantities here treated as small. But the size of the hexagons (under the boundary conditions postulated) is determinate, at any rate when they assert themselves early enough.

An appendix deals with a related analytical problem having various physical interpretations, such as the symmetrical vibration in two dimensions of a layer of air enclosed by a nearly circular wall.

The general Eulerian equations of fluid motion are in the usual notation :-

$\frac{\mathrm{D} u}{\mathrm{D} t}=\mathrm{X}-\frac{1}{\rho} \frac{d p}{d x}, \quad \overline{\mathrm{D} v}=\mathrm{Y}-\frac{1}{\rho} \frac{d p}{d y}, \quad \frac{\mathrm{D} w}{\mathrm{D} t}=\mathrm{Z}-\frac{1}{\rho} \frac{d p}{d z},$.

where

$$
\frac{\mathrm{D}}{\mathrm{D} t}=\frac{d}{d t}+u \frac{d}{d x}+v \frac{d}{d y}+w \frac{d}{d z}, \quad . \quad .
$$

and $\mathrm{X}, \mathrm{Y}, \mathrm{Z}$ are the components of extraneous force reckoned per unit of mass. If, neglecting viscosity, we suppose that gravity is the only impressed force,

$$
\mathrm{X}=0, \quad \mathrm{Y}=0, \quad \mathrm{Z}=-g, \quad . \quad .
$$

$z$ being measured upwards. In equations (1) $\rho$ is variable in consequence of variable temperature and variable pressure. But, as Boussinesq * has shown, in the class of problems under consideration the influence of pressure is unimportant and even the variation with temperature may be disregarded except in so far as it modifies the operation of grarity. If we write $\rho=\rho_{0}+\delta \rho$, we have

$$
g \rho=g \rho_{0}\left(1+\delta \rho / \rho_{0}\right)=g \rho_{0}-g \rho_{0} \alpha \theta
$$

where $\theta$ is the temperature reckoned from the point where $\rho=\rho_{0}$ and $\alpha$ is the coefficient of expansion. We may now identify $\rho$ in (1) with $\rho_{0}$, and our equations become

$$
\frac{\mathrm{D} u}{\mathrm{D} t}=-\frac{1}{\rho} \frac{d \mathrm{P}}{d x}, \quad \frac{\mathrm{D} v}{\overline{\mathrm{D}} t}=-\frac{1}{\rho} \frac{d \mathrm{P}}{d y}, \quad \frac{\mathrm{D} w}{\mathrm{D} t}=-\frac{1}{\rho} \frac{d \mathrm{P}}{d z}+\gamma \theta, .
$$

wher $\rho$ is a constant, $\gamma$ is written for $g \alpha$, and $\mathrm{P}$ for $p+g \rho z$.

* Theorie Analytique de la Chaleur, t. ii. p. 172 (1903). 
Also, since the fluid is now treated as incompressible,

$$
\frac{d u}{d \boldsymbol{x}}+\frac{d v}{d y}+\frac{d w}{d z}=0 . \quad \text {. . . . . . }
$$

The equation for the conduction of heat is,

$$
\overline{\mathrm{D} \theta}=\kappa\left(\frac{d^{2} \theta}{d x^{2}}+\frac{d^{2} \theta}{d y^{2}}+\frac{d^{2} \theta}{d z^{2}}\right), \ldots . .
$$

in which $\kappa$ is the diffusibility for temperature. These are the equations employed by Boussinesq.

In the particular problems to which we proceed the fluid is supposed to be bounded by two infinite fixed planes at $z=0$ and $z=\zeta$, where also the temperatures are maintained constant. In the equilibrium condition $u, v, w$ vanish and $\theta$ being a function of $z$ only is subject to $d^{2} \theta / d z^{2}=0$, or $d \theta / d z=\beta$, where $\beta$ is a constant representing the temperature gradient. If the equilibrium is stable, $\beta$ is positive; and if unstable with the higher temperature below, $\beta$ is negative. It will be convenient, however, to reckon $\theta$ as the departure from the equilibrium temperature $\Theta$. The only change required in equations (4) is to write $\approx$ for $P$, where

$$
\varpi=\mathrm{P}-\rho \gamma \int \Theta d z . \quad . \quad . \quad . \quad .
$$

In equation (6) $\mathrm{D} \theta / \mathrm{D} t$ is to be replaced by $\mathrm{D} \theta / \mathrm{D} t+u \beta$.

The question with which we are principally concerned is the effect of a small departure from the condition of equilibrium, whether stable or unstable. For this purpose it suffices to suppose $u, v, w$, and $\theta$ to be small. When we neglect the squares of the small quantities, $\mathrm{D} / \mathrm{D} t$ identifies itself with $d / d t$ and we get

$$
\begin{array}{r}
\frac{d u}{d t}=-\frac{1}{\rho} \frac{d \varpi}{d x}, \quad \frac{d v}{d t}=-\frac{1}{\rho} \frac{d \varpi}{d y}, \quad \frac{d w}{d t}=-\frac{1}{\rho} \frac{d \varpi}{d z}+\gamma \theta, . \\
\frac{d \theta}{d t}+\beta w=\kappa\left(\frac{d^{2} \theta}{d x^{2}}+\frac{d^{2} \theta}{d y^{2}}+\frac{d^{2} \theta}{d z^{2}}\right), \ldots .
\end{array}
$$

which with (5) and the initial and boundary conditions suffice for the solution of the problem. The boundary conditions are that $u=0, \theta=0$, when $z=0$ or $\zeta$.

We now assume in the usual manner that the small quantities are proportional to

$$
e^{i l x} e^{i m y} e^{n t}, \quad . \quad . \quad . \quad .
$$


so that $(8),(5),(9)$ become

$$
\begin{array}{r}
n u=-\frac{i l \varpi}{\rho}, \quad n v=-\frac{i m \varpi}{\rho}, \quad n w=-\frac{1}{\rho} \frac{d \varpi}{d z}+\gamma \theta, . \\
i l u+i m v+d w / d z=0, . \quad . \quad . \\
n \theta+\beta w=\kappa\left(d^{2} / d z^{2}-l^{2}-m^{2}\right) \theta, . \quad . \quad .
\end{array}
$$

from which by elimination of $u, v, w$, we derive

$$
\frac{n}{l^{2}+m^{2}} \frac{d^{2} w}{d z^{2}}=n w-\gamma \theta \text {. . . . . }
$$

Having regard to the boundary conditions to be satisfied by $w$ and $\theta$, we now assume that these quantities are proportional to $\sin s z$, where $s=q \pi / \zeta$, and $q$ is an integer. Hence

$$
\begin{gathered}
\beta w+\left\{n+\kappa\left(l^{2}+m^{2}+s^{2}\right)\right\} \theta=0, \quad . \quad . \\
n\left(l^{2}+m^{2}+s^{2}\right) w-\gamma\left(l^{2}+m^{2}\right) \theta=0,
\end{gathered}
$$

and the equation determining $n$ is the quadratic

$$
n^{2}\left(l^{2}+m^{2}+s^{2}\right)+n \kappa\left(l^{2}+m^{2}+s^{2}\right)^{2}+\beta \gamma\left(l^{2}+m^{2}\right)=0 .
$$

When $\kappa=0$, there is no conduction, so that each element of the fluid retains its temperature and density. If $\beta$ be positive, the equilibrium is stable, and

$$
n=\frac{ \pm i \sqrt{ }\left\{\beta \gamma\left(l^{2}+m^{2}\right)\right\}}{\sqrt{ }\left\{l^{2}+m^{2}+s^{2}\right\}}, \text {. . . . . }
$$

indicating vibrations about the condition of equilibrium. If, on the other hand, $\beta$ be negative, say $-\beta^{\prime}$,

$$
n=\frac{ \pm \sqrt{ }\left\{\beta^{\prime} \gamma\left(l^{2}+m^{2}\right)\right\}}{\sqrt{ }\left\{l^{2}+m^{2}+s^{2}\right\}} \text {. . . . . }
$$

When $n$ has the positive value, the corresponding disturbance increases exponentially with the time.

For a given value of $l^{2}+m^{2}$, the numerical values of $n$ diminish without limit as $s$ increases-that is, the more subdivisions there are along $z$. The greatest value corresponds with $q=1$ or $s=\pi / \zeta$. On the other hand, if $s$ be given, $|n|$ increases from zero as $l^{2}+m^{2}$ increases from zero (great wave-lengths along $x$ and $y$ ) up to a finite limit when $l^{2}+m^{2}$ is large (small wave-lengths along $x$ and $y$ ). This case of 
no conductivity falls within the scope of a former investigation where the fluid was supposed from the beginning to be incompressible but of variable density *.

Returning to the consideration of a finite conductivity, we have again to distinguish the cases where $\beta$ is positive and negative. When $\beta$ is negative (higher temperature below) both values of $n$ in (17) are real and one is positive. The equilibrium is unstable for all values of $l^{2}+m^{2}$ and of $s$. If $\beta$ be positive, $n$ may be real or complex. In either case the real part of $n$ is negative, so that the equilibrium is stable whatever $l^{2}+m^{2}$ and $s$ may be.

When $\beta$ is negative $\left(-\beta^{\prime}\right)$, it is important to inquire for what values of $l^{2}+m^{2}$ the instability is greatest, for these are the modes which more and more assert themselves as time elapses, even though initially they may be quite subordinate. That the positive value of $n$ must have a maximum appears when we observe it tends to vanish both when $l^{2}+m^{2}$ is small and also when $l^{2}+m^{2}$ is large. Setting for shortness $l^{2}+m^{2}+s^{2}=\sigma$, we may write (17)

$$
n^{2} \sigma+n \kappa \sigma^{2}-\beta^{\prime} \gamma\left(\sigma-s^{2}\right)=0,
$$

and the question is to find the value of $\sigma$ for which $n$ is greatest, $s$ being supposed given. Making $d n / d \sigma=0$, we get on differentiation

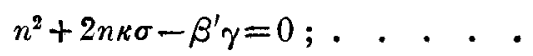

and on elimination of $n^{2}$ between (20), (21)

$$
n=\frac{\beta^{\prime} \gamma s^{2}}{\kappa \sigma^{2}} \cdot \text {. . . . . . }
$$

Using this value of $n$ in (21), we find as the equation for $\sigma$

$$
\frac{2 s^{2}}{\sigma}=1-\frac{\beta^{\prime} \gamma s^{4}}{\kappa^{2} \sigma^{4}} \text {. . . . . . }
$$

When $\kappa$ is relatively great, $\sigma=2 s^{2}$, or

$$
l^{2}+m^{2}=s^{2} \text {. . . . . . }
$$

A second approximation gives

$$
l^{2}+m^{2}=s^{2}+\frac{\beta^{\prime} \gamma}{8 \kappa^{2} s^{2}} . \text {. . . . . }
$$

The corresponding value of $n$ is

$$
n=\frac{\beta^{\prime} \gamma}{4 \kappa s^{2}}\left\{1-\frac{\beta^{\prime} \gamma}{8 \kappa^{2} s^{4}}\right\} . . . .
$$

* Proc. Lond. Math. Soc. vol. xiv. p. 170 (1883) ; Scientific Papers, rol, ii. p. 200 , 
The modes of greatest instability are those for which $s$ is smallest, that is equal to $\pi / \zeta$, and

$$
l^{2}+m^{2}=\frac{\pi^{2}}{\zeta^{2}}+\frac{\beta^{\prime} \gamma}{8 \kappa^{2} \pi^{2} / \zeta^{2}} . \quad . \quad . \quad . .
$$

For a two-dimersional disturbance we may make $m=0$ and $l=2 \pi / \lambda$, where $\lambda$ is the wave-length along $x$. The $\lambda$ of maximum instability is thus approximately

$$
\lambda=2 \zeta . \text {. . . . , . }
$$

Again, if $l=m=2 \pi / \lambda$, as for supare cells,

$$
\lambda=2 \sqrt{ } 2 . \zeta, \cdot . \cdot . \cdot
$$

greater than before in the ratio $\sqrt{ } 2: 1$.

We have considered especially the cases where $\kappa$ is relatively small and relatively large. Intermediate cases would need to be dealt with by a numerical solution of (23).

When $w$ is known in the form

$$
w=W e^{i l x} e^{i m y} \sin s z . e^{n t}, \quad . \quad . \quad .
$$

$n$ being now a known function of $l, m, s, u$ and $v$ are at once derived by means of (11) and (12). 'Thus

$$
u=\frac{i l}{l^{2}+m^{2}} \frac{d w}{d z}, \quad v=\frac{i m}{l^{2}+m^{2}} \frac{d w}{d z} . . .
$$

The connexion between $w$ and $\theta$ is given by (15) or (16). When $\beta$ is negative and $n$ positive, $\theta$ and $w$ are of the same sign.

As an example in two dimensions of (30), (31), we might have in real form

$$
\begin{gathered}
w=W \cos x \cdot \sin z \cdot e^{n t}, \quad . \quad . \quad . \\
u=-W \sin x \cdot \cos z \cdot e^{n t}, \quad v=0 .
\end{gathered}
$$

Hitherto we have supposed the fluid to be destitute of viscosity. When we include viscosity, we must add $\nu\left(\nabla^{2} u, \nabla^{2} v, \nabla^{2} w\right)$ on the right of equations (1), (8), and (11), $v$ being the kinematic coefficient. Equations (12) and (13) remain unaffected. And in (11)

$$
\nabla^{2}=d^{2} / d z^{2}-l^{2}-m^{2} .
$$

We have also to reconsider the boundary conditions at $z=0$ and $z=\zeta$. We may still suppose $\theta=0$ and $w=0$; but for a further condition we should probably prefer $d w / d z=0$, corresponding to a fixed solid wall. But this entails much complication, and wo may content ourselves with the 
supposition $d^{2} w / d z^{2}=0$, which (with $w=0$ ) is satisfied by taking as before $w$ proportional to $\sin s z$ with $s=q \pi / \zeta$. This is equivalent to the annulment of lateral forces at the wall. For (Lamb's 'Hydrodynanics,' $\$ 323,326$ ) these forces aro expressed in general by

$$
p_{x z}=\frac{d w}{d x}+\frac{d u}{d z}, \quad p_{y z}=\frac{d w}{d y}+\frac{d v}{d z}, . . .
$$

while here $w=0$ at the boundaries requires also $d w / d x=0$, $d w / d y=0$. Hence, at the boundaries, $d^{2} u / d x d z, d^{2} v / d y d z$ vanish, and therefore by $(5), d^{2} w / d z^{2}$.

Equation (15) remains unaltered :-

$$
\beta w+\left\{n+\kappa\left(l^{2}+m^{2}+s^{2}\right)\right\} \theta=0, . .
$$

and (16) becomes

$$
\left\{n+\nu\left(l^{2}+m^{2}+s^{2}\right)\right\}\left(l^{2}+m^{2}+s^{2}\right) w-\gamma\left(l^{2}+m^{2}\right) \theta=0 .
$$

Writing as before $\sigma=l^{2}+m^{2}+s^{2}$, we get the equation in $n$

$$
(n+\kappa \sigma)(n+\nu \sigma) \sigma+\beta \gamma\left(l^{2}+m^{2}\right)=0, \quad .
$$

which takes the place of $(1 \bar{i})$.

If $\gamma=0$ (no expansion with heat) the equations degrade, and we have two simple alternatives. In the first $n+\kappa \sigma=0$ with $w=0$, signifying conduction of heat with no motion. In the second $n+\nu \sigma=0$, when the relation between $w$ and $\theta$ becomes

$$
\beta w+\sigma(\kappa-\nu) \theta=0 . . . . .
$$

In both cases, since $n$ is real and negative, the disturbance is stable.

If we neglect $\kappa$ in (37), the equation takes the same form $(20)$ as that already considered when $\nu=0$. Hence the results expressed in (22), (23), (24), (25), (26), (27) are applicable with simple substitution of $\nu$ for $\kappa$.

In the general equation (37) if $\beta$ be positive, as $\gamma$ is supposed always to be, the values of $n$ may be real or complex. If real they are both negative, and if complex the real part is negative. In either case the disturbance dies down. As was to be expected, when the temperature is higher above, the equilibrium is stable.

In the contrary case when $\beta$ is negative $\left(-\beta^{\prime}\right)$ the roots of the quadratic are always real, and one at least is negative. There is a positive root only when

$$
\beta^{\prime} \gamma\left(l^{2}+m^{2}\right)>\kappa \nu \sigma^{3} \text {. . . . . }
$$


If $\kappa$, or $\nu$, vanish there is instability; but if $\kappa$ and $\nu$ are finite and large enough, the equilibrium for this disturbance is stable, although the higher temperature is underneath.

Inequality (39) gives the condition of instability for the particular disturbance $(l, m, s)$. It is of interest to inquire at what point the equilibrium becomes unstable when there is no restriction upon the value of $l^{2}+m^{2}$. In the equation

$$
\beta^{\prime} \gamma\left(l^{2}+m^{2}\right)-\kappa \nu \sigma^{3}=\beta^{\prime} \gamma\left(\sigma-s^{2}\right)-\kappa \nu \sigma^{3}=0, .
$$

we see that the left-hand member is negative when $l^{2}+m^{2}$ is small and also when it is large. When the conditions are such that the equation can only just be satisfied with some value of $l^{2}+m^{2}$, or $\sigma$, the derived equation

$$
\beta^{\prime} \gamma-3 \kappa \nu \sigma^{2}=0 . \text {. . . . . }
$$

must also hold good, so that

and

$$
\sigma=3 s^{2} / \mathbf{2}, \quad l^{2}+m^{2}=\frac{1}{2} s^{2}, \quad . \quad .
$$

$$
\beta^{\prime} \gamma=27 \kappa \nu s^{4} / 4 \text {. . . . . . . }
$$

Unless $\beta^{\prime} \gamma$ exceeds the value given in (43) there is no instability, however $l$ and $m$ are chosen. But the equation still contains $s$, which may be as large as we please. The smallest value of $s$ is $\pi / \zeta$. The condition of instability when $l, m$, and $s$ are all unrestricted is accordingly

$$
\beta^{\prime} \gamma>\frac{27 \pi^{4} \kappa \nu}{4 \zeta^{4}} \ldots . . . . .
$$

If $\beta^{\prime} \gamma$ falls below this amount, the equilibrium is altogether stable. I am not aware that the possibility of complete stability under such circumstances has been contemplated.

To interpret (44) more conveniently, we may replace $\beta^{\prime}$ by $\left(\Theta_{2}-\Theta_{1}\right) / \zeta$ and $\gamma$ by $g\left(\rho_{2}-\rho_{1}\right) / \rho_{1}\left(\Theta_{2}^{*}-\Theta_{1}\right)$, so that

$$
\beta^{\prime} \gamma=\frac{g}{\zeta} \frac{\rho_{2}-\rho_{1}}{\rho_{1}}, \ldots . . . . .
$$

where $\Theta_{2}, \Theta_{1}, \rho_{2}$, and $\rho_{1}$ are the extreme temperatures and densities in equilibrium. Thus (44) becomes

$$
\frac{\rho_{2}-\rho_{1}}{\rho_{1}}>\frac{27 \pi^{4} \kappa \nu}{4 g \zeta^{3}} \cdot \text {. . . . . }
$$

In the case of air at atmospheric conditions we may take in C.G.S. measure

$$
\nu=\cdot 14, \quad \text { and } \quad \kappa={ }_{2}^{5} \nu \text { (Maxwell's Theory). }
$$


Also $g=980$, and thus

$$
\frac{\rho_{2}-\rho_{1}}{\rho_{1}} \cdot \frac{\cdot 033}{\zeta^{3}} . . . . . . .
$$

For example, if $\zeta=1 \mathrm{~cm}$, instability requires that the density at the top exceed that at the bottom by one-thirtieth part, corresponding to about $9^{\circ} \mathrm{C}$. of temperature. We should not forget that our method postulates a small value of $\left(\rho_{2}-\rho_{1}\right) / \rho_{1}$. Thus if $\kappa \nu$ be given, the application of (46) may cease to be legitimate unless $\zeta$ be large enough.

It may be remarked that the influence of viscosity would be increased were we to suppose the horizontal velocities (instead of the horizontal forces) to be annulled at the boundaries.

The problem of determining for what value of $l^{2}+m^{2}$, or $\sigma$, the instability, when finite, is a maximum is more complicated. The differentiation of (37) with respect to $\sigma$ gives

whence

$$
n^{2}+2 n \sigma(\kappa+\nu)+3 \kappa \nu \sigma^{2}-\beta^{\prime} \gamma=0, . . .
$$

$$
n=\frac{\beta^{\prime} \gamma s^{2}-2 \kappa \nu \sigma^{3}}{\sigma^{2}(\kappa+\nu)}, \quad . \quad . \quad . .
$$

expressing $n$ in terms of $\sigma$. To find $\sigma$ we have to eliminate $n$ between (44) and (45). The result is

$$
\sigma^{6} \kappa \nu(\kappa-\nu)^{2}+\sigma^{4} \beta^{\prime} \gamma(\kappa+\nu)^{2}-\sigma^{3} \cdot 2 \beta^{\prime} \gamma s^{2}\left(\kappa^{2}+\nu^{2}\right)-\beta^{2} \gamma^{2} s^{4}=0,
$$

from which, in particular cases, $\sigma$ could be found by numerical computation. From (50) we fall back on (23) by supposing $\nu=0$, and again on a similar equation if we $\operatorname{suppos} \theta \kappa=0$.

But the case of a nearly evanescent $n$ is probably the more practical. In an experiment the teniperature gradient could not be established all at once and we may suppose the progress to be very slow. In the earlier stages the equilibrium would be stable, so that no disturbance of importance would occur until $n$ passed throngh zero to the positive side, corresponding to (44) or (46). The breakdown thus occurs for $s=\pi / \zeta$, and by (42) $l^{2}+m^{2}=\pi^{2} / 2 \zeta^{2}$. And since the evanescence of $n$ is equivalent to the omission of $d / d t$ in the original equations, the motion thus determined has the character of a steady motion. The constant multiplier is, however, arbitrary; and there is nothing to determine it so long as the squares of $u, v, w, \theta$ are neglected. 
In a particular solution where $w$ as a function of $x$ and $y$ has the simplest form, say

$$
w=2 \cos x \cdot \cos y, \text {. . . . . }
$$

the particular coefficients of $x$ and $y$ which enter have relation to the particular axos of reference employed. If we rotate these axes through an angle $\phi$, we have

$$
\begin{aligned}
w & =2 \cos \left\{x^{\prime} \cos \phi-y^{\prime} \sin \phi\right\} \cdot \cos \left\{x^{\prime} \sin \phi+y^{\prime} \cos \phi\right\} \\
& =\cos \left\{x^{\prime}(\cos \phi-\sin \phi)\right\} \cdot \cos \left\{y^{\prime}(\cos \phi+\sin \phi)\right\} \\
& +\sin \left\{x^{\prime}(\cos \phi-\sin \phi)\right\} \cdot \sin \left\{y^{\prime}(\cos \phi+\sin \phi)\right\} \\
& +\cos \left\{x^{\prime}(\cos \phi+\sin \phi)\right\} \cdot \cos \left\{y^{\prime}(\cos \phi-\sin \phi)\right\} \\
& -\sin \left\{x^{\prime}(\cos \phi+\sin \phi)\right\} \cdot \sin \left\{y^{\prime}(\cos \phi-\sin \phi)\right\} .
\end{aligned}
$$

For example, if $\phi=\frac{1}{4} \pi,(52)$ becomes

$$
w=\cos \left(y^{\prime} \sqrt{ } 2\right)+\cos \left(x^{\prime} \sqrt{ } 2\right) . . . .
$$

It is to be observed that with the general value of $\phi$, if we call the coefficients of $x^{\prime}, y^{\prime}, l$ and $m$ respectively, we have in every part $l^{2}+m^{2}=2$, unaltered from the original value in (51).

The character of $w$, under the condition that all the elementary terms of which it is composed are subject to $l^{2}+m^{2}=$ constant $\left(k^{2}\right)$, is the same as for the transverse displacement of an infinite stretched membrane, vibrating with one definite frequency. The limitation upon $w$ is, in fact, merely that it satisfies

$$
\left(d^{2} / d x^{2}+d^{2} / d y^{2}+k^{2}\right) w=0 . \text {. . . }
$$

The character of $w$ in particular solutions of the membrane problem is naturally associated with the nodal system $(w=0)$, where the membrane may be regarded as held fast ; and we may suppose the nodal system to divide the plane into similar parts or cells, such as squares, equilateral triangles, or regular hexagons. But in the present problem it is perhaps more appropriate to consider divisions of the plane with respect to which $w$ is symmetrical, so that $d w / d n$ is zero on the straight lines forming the divisions of the cells. The more natural analogy is then with the twodimensional vibration of air, where $w$ represents velocitypotential and the divisions may be regarded as fixed walls.

The simplest case is, of course, that in which the cells are 
squares. If the sides of the squares be $2 \pi$, we may take with axes parallel to the sides and origin at centre

$$
w=\cos x+\cos y, \quad \text {. . . . }
$$

being thus composed by superposition of two parts for each of which $k^{2}=1$. This makes $d w / d x=-\sin x$, vanishing when $x= \pm \pi$. Similarly, $d w / d y$ vanishes when $y= \pm \pi$, so that the sides of the square behave as fixed walls. To find the places where $w$ changes sign, we write it in the form

$$
w=2 \cos \frac{x+y}{2} \cdot \cos \frac{x-y}{2}, \text {. . . . }
$$

giving $x+y= \pm \pi, x-y= \pm \pi$, lines which constitute the inscribed square (fig. 1). Within this square $w$ has one sign (say + ) and in the four rightangled triangles left over the - sign. When the whole plane is considered, there is no want of symmetry between the + and the - regions.

The principle is the same when the elementary cells are equilateral triangles or hexagons; but I am not aware that an analytical solution has been obtained for these cases. An experimental determination of $k^{2}$ might be made by observing the time of vibration under gravity of water contained in a trough with vertical sides and of corresponding section, which depends upon the same differential equation and boundary conditions*. The particular vibration in question is not the slowest possible, but that where there is a simultaneous rise at the centre and fall at the walls all round, with but one curve of zero elevation between.

In the case of the hexagon, we may regard it as deviating comparatively little from the circular form and employ the approximate methods then applicable. By an argument analogous to that formerly developed $\dagger$ for the boundary condition $w=0$, we may convince ourselves that the value of $k^{2}$ for the hexagon cannot differ much from that appropriate to a circle of the same area. Thus if $a$ be the radius

* See Phil. Mag. vol. i. p. 257 (1876); Scientific Papers, vol. i. pp. 265,271 .

† 'Theory of Sound, $\$ 209$; compare also $\$ 317$. See Appendix. 
of this circle, $k$ is given by $J_{0}^{\prime}(k a)=0, J_{0}$ being the Bessel's function of zero order, or $k a=3.832$. If $b$ be the side of the hexagon, $a^{2}=3 \sqrt{ } 3 \cdot b^{2} / 2 \pi$.

\section{APPENDIX.}

On the nearly symmetrical solution for a nearly circular area, when $w$ satisfies $\left(d^{2} / d x^{2}+d^{2} / d y^{2}+k^{2}\right) w=0$ and makes $d w / d n=0$ on the boundary.

Starting with the true circle of radius $a$, we have $w$ a function of $r$ (the radius vector) only, and the solution is $w=\mathrm{J}_{0}(k r)$ with the condition $\mathrm{J}_{0}^{\prime}(k a)=0$, yielding $k a=3 \cdot 832$, which determines $k$ if $a$ be given, or $a$ if $k$ be given. In the problem proposed the boundary is only approximately circular, so that we write $r=a+\rho$, where $a$ is the mean value and

$$
\rho=\alpha_{1} \cos \theta+\beta_{1} \sin \theta+\ldots+\alpha_{n} \cos n \theta+\beta_{n} \sin n \theta .
$$

In (57) $\theta$ is the vectorial angle and $\alpha_{1}$ \&c. are quantities small relatively to $a$. The general solution of the differential equation being

$$
\begin{aligned}
w=\mathrm{A}_{0} \mathrm{~J}_{0}(k r)+\mathrm{J}_{1}(k r)\left\{\mathrm{A}_{1} \cos \theta+\mathrm{B}_{1} \sin \theta\right\} & \\
& +\ldots+\mathrm{J}_{n}(k r)\left\{\mathrm{A}_{n} \cos n \theta+\mathrm{B}_{n} \sin \theta\right\}, . .
\end{aligned}
$$

we are to suppose now that $A_{1}$, \&c., are small relatively to $A_{0}$. It remains to consider the boundary condition.

If $\phi$ denote the small angle between $r$ and the normal $d n$ measured outwards,

and

$$
\frac{d w}{d n}=\frac{d w}{d r} \cos \phi-\frac{d w}{r d \theta} \sin \phi, \ldots . . .
$$

$$
\tan \phi=\frac{d r}{r d \theta}=\frac{d \rho}{a d \theta}=\frac{n}{\alpha}\left(-\alpha_{n} \sin n \theta+\beta_{n} \cos n \theta\right)
$$

with sufficient approximation, only the general term being written. In formulating the boundary condition $d w / d n=0$ correct to the second order of small quantities, we require $d w / d r$ to the second order, but $d w / d \theta$ to the first order only. We have

$$
\begin{gathered}
\frac{1}{k} \frac{d w}{d r}=\mathrm{A}_{0}\left\{\mathrm{~J}_{0}^{\prime}(k a)+k \rho \mathrm{J}_{0}^{\prime \prime}(k a)+\frac{1}{2} k^{2} \rho^{2} \mathrm{~J}_{0}^{\prime \prime \prime}(k a)\right\} \\
+\left\{\mathrm{J}_{n}^{\prime}(k a)+k \rho \mathrm{J}_{n}^{\prime \prime}(k a)\right\}\left\{\mathrm{A}_{n} \cos n \theta+\mathrm{B}_{n} \sin n \theta\right\} \\
\frac{d w}{a-d \theta}=\frac{n}{a} \mathrm{~J}_{n}(k a)\left\{-\mathrm{A}_{n} \sin n \theta+\mathrm{B}_{n} \cos n \theta\right\}
\end{gathered}
$$


544 Lord Rayleigh on Convection Currents in

and for the boundary condition, setting $k a=z$ and omitting the argument in the Bessel's functions,

$$
\begin{aligned}
& \mathrm{A}_{0}\left\{\mathrm{~J}_{0}{ }^{\prime} \cdot \cos \phi+k \rho \mathrm{J}_{0}{ }^{\prime \prime}+\frac{1}{2} k^{2} \rho^{2} \mathrm{~J}_{0}{ }^{\prime \prime \prime}\right\} \\
& +\left\{\mathrm{J}_{n}{ }^{\prime}+k \rho \mathrm{J}_{n}{ }^{\prime \prime}\right\}\left\{\mathrm{A}_{n} \cos n \theta+\mathrm{B}_{n} \sin n \theta\right\} \\
& -\frac{n^{2}}{a z} \mathrm{~J}_{n}\left\{-\mathrm{A}_{n} \sin n \theta+\mathrm{B}_{n} \cos n \theta\right\}\left\{-\alpha_{n} \sin n \theta\right. \\
& \left.\quad+\beta_{n} \cos n \theta\right\}=0 . .
\end{aligned}
$$

If for the moment we omit the terms of the second order, we have

$$
\begin{aligned}
\mathrm{A}_{0} J_{0}{ }^{\prime}+k \mathrm{~A}_{0} J_{0}{ }^{\prime \prime}\left\{\alpha_{n} \cos n \theta+\beta_{n} \sin n \theta\right\} \\
+J_{n}\left\{\mathrm{~A}_{n} \cos n \theta+\mathrm{B}_{n} \sin n \theta\right\}=0 ; .
\end{aligned}
$$

so that

$$
\mathrm{J}_{0}^{\prime}(z)=0, \quad \text { and }
$$

$$
k \mathrm{~A}_{0} \mathrm{~J}_{0}{ }^{\prime \prime} \cdot \alpha_{n}+\mathrm{J}_{n}{ }^{\prime} \cdot \mathrm{A}_{n}=0, \quad k \mathrm{~A}_{0} \mathrm{~J}_{0}{ }^{\prime \prime} \cdot \beta_{n}+\mathrm{J}_{n}{ }^{\prime} \cdot \mathrm{B}_{n}=0 .
$$

To this order of approximation $z,=k a$, has the same value as when $\rho=0$; that is to say, the equivalent radius is equal to the mean radius, or (as we may also express it) $k$ may be regarded as dependent upon the area only. Equations (6:3) determine $\mathrm{A}_{n}, \mathrm{~B}_{n}$ in terms of the known quantities $\alpha_{n}, \beta_{n}$.

Since $J_{0}^{\prime}$ is a small quantity, $\cos \phi$ in (61) may now be omitted. To obtain a corrected evaluation of $z$, it suffices to take the mean of (61) for all values of $\theta$. Thus

$$
\begin{aligned}
\mathrm{A}_{0}\left\{2 \mathrm{~J}_{0}{ }^{\prime}+\frac{1}{2} k^{2} \mathrm{~J}_{0}^{\prime \prime \prime}\right. & \left.\left(\alpha_{n}{ }^{2}+\beta_{n}{ }^{2}\right)\right\} \\
& +\left\{k \mathrm{~J}_{n}{ }^{\prime \prime}-n^{2} J_{n} / a z\right\}\left\{\alpha_{n} \mathrm{~A}_{n}+\beta_{n} \mathrm{~B}_{n}\right\}=0,
\end{aligned}
$$

or on substitution of the approximate values of $\mathrm{A}_{n}, \mathrm{~B}_{n}$ from (63),

$$
\mathrm{J}_{0}^{\prime}=\frac{1}{2} k^{2}\left(\alpha_{n}{ }^{2}+\beta_{n}{ }^{2}\right)\left\{\frac{J_{0}^{\prime \prime}}{J_{n}{ }^{\prime}}\left(J_{n}{ }^{\prime \prime}-\frac{n^{2} J_{n}}{z^{2}}\right)-\frac{J_{0}{ }^{\prime \prime \prime}}{z}\right\} .
$$

This expression may, however, be much simplified. In virtue of the general equation for $J_{n}$,

$$
\mathrm{J}_{n}^{\prime \prime}-\frac{n^{2}}{z^{2}} \mathrm{~J}_{n}=-\frac{\mathrm{J}_{n}^{\prime}}{z}-\mathrm{J}_{n} ;
$$

and since here ${ }^{-} J_{0}^{\prime}=0$ approximately,

Thus

$$
\mathrm{J}_{0}^{\prime \prime}=-\mathrm{J}_{0}, \quad \mathrm{~J}_{0}^{\prime \prime \prime}=-z^{-1} \mathrm{~J}_{0}^{\prime \prime}=z^{-1} \mathrm{~J}_{0} .
$$

$$
\mathrm{J}_{0}^{\prime}(z)=\frac{1}{2} k^{2} \mathrm{~J}_{0} \cdot \Sigma\left(\boldsymbol{\alpha}_{n}{ }^{2}+\beta_{n}{ }^{2}\right)\left\{\frac{J_{n}}{J_{n}{ }^{\prime}}+\frac{1}{2 z}\right\}, \quad .
$$

the sign of summation with respect to $n$ being introduced. 
Let us now suppose that $a+d a$ is the equivalent radius, so that $J_{0}^{\prime}(k a+k d a)=0$, that is the radius of the exact circle which corresponds to the value of $k$ appropriate to the approximate circle. Then

and

$$
J_{0}^{\prime}(z)+k d a J_{0}^{\prime \prime}(z)=0,
$$

$$
d a=-\frac{J_{0}^{\prime}}{k J_{0}{ }^{\prime \prime}}=k \Sigma\left(\alpha_{n}{ }^{2}+\beta_{n}{ }^{2}\right)\left\{\frac{J_{n}}{2 J_{n}{ }^{\prime}}+\frac{1}{4 z}\right\} .
$$

Again, if $a+d a^{\prime}$ be the radius of the true circle which has the same area as the approximate circle

and

$$
d a^{\prime}=\frac{1}{4 a} \Sigma\left(\alpha_{n}^{2}+\beta_{n}{ }^{2}\right), \quad \text {. . . . }
$$

$$
d a^{\prime}-d a=-\Sigma \frac{\alpha_{n}{ }^{2}+\beta_{n}{ }^{2}}{2 a} \frac{z \mathrm{~J}_{n}(z)}{2 J_{n}{ }^{\prime}(z)}, \quad . \quad .
$$

where $z$ is the first root (after zero) of $\mathrm{J}_{0}{ }^{\prime}(z)=0$, viz. $3 \cdot 832$.

The question with which we are mainly concerned is the sign of $d a^{\prime}-d a$ for the various values of $n$. When $n=1$, $\mathrm{J}_{1}(z)=-J_{0}{ }^{\prime}(z)=0$, so that $d a=d a^{\prime}$, a result which was to be expected, since the terms in $\alpha_{1}, \beta_{1}$ represent approximately a displacement merely of the circle, without alteration of size or shape. We will now examine the sign of $J_{n} / J_{n}{ }^{\prime}$ when $n=2$, and 3 .

For this purpose we may employ the sequence equations

$$
\mathrm{J}_{n+1}=\frac{2 n}{z} \mathrm{~J}_{n}-\mathrm{J}_{n-1}, \quad \mathrm{~J}_{n}{ }^{\prime}=\frac{1}{2} \mathrm{~J}_{n-1}-\frac{1}{2} \mathrm{~J}_{n+1},
$$

which allow $J_{n}$ and $J_{n}{ }^{\prime}$ to be expressed in terms of $J_{1}$ and $J_{0}$, of which the former is here zero. We find

$$
\begin{array}{lll}
\mathrm{J}_{2}=-\mathrm{J}_{0}, & \mathrm{~J}_{2}=-4 z^{-1} \mathrm{~J}_{0}, & \mathrm{~J}_{4}=\left(1-24 z^{-2}\right) \mathrm{J}_{0} ; \\
\mathrm{J}_{1}{ }^{\prime}=\mathrm{J}_{0}, & \mathrm{~J}_{2}{ }^{\prime}=2 z^{-1} \mathrm{~J}_{0}, & \mathrm{~J}_{3}^{\prime}=\left(12 z^{-2}-1\right) \mathrm{J}_{0} .
\end{array}
$$

Thus

$$
\frac{J_{1}}{J_{1}^{\prime}}=0, \quad \frac{J_{2}}{J_{2}^{\prime}}=-\frac{z}{2}, \quad \frac{J_{3}}{J_{3}^{\prime}}=\frac{4 z}{z^{2}-12} ;
$$

whence on introduction of the actual value of $z$, viz. $3 \cdot 832$, we see that $J_{2} / J_{2}{ }^{\prime}$ is negative, and that $J_{3} / J_{3}{ }^{\prime}$ is positive.

When $n>z$, it is a general proposition that $J_{n}(z)$ and $\mathrm{J}_{n}{ }^{\prime}(z)$ are both positive ${ }^{*}$. Hence for $n=4$ and onwards, $\mathrm{J}_{n} / \mathrm{J}_{n}{ }^{\prime}$ is positive when $z=3 \cdot 832$. We thus arrive at the

* See, for example, Theory of Sound, $\$ 210$.

Phil. Mag. S. 6. Vol. 32. No. 192. Dec. 1916. 
curious conclusion that when $n=2, d a^{\prime}>d a$, as happens for all values of $n$ (exceeding unity) when the boundary condition is $w=0$, but that when $n>2, d a^{\prime}<d a$. The existence of the exceptional case $n=2$ precludes a completely general statement of the effect of a departure from the truly circular form ; but if the terms for which $n=2$ are absent, as they would be in the case of any regular polygon with an even number of sides, regarded as a deformed circle, we may say that $d a^{\prime}<d a$. In the physical problems the effect of a departure from the circular form is then to depress the pitch when the area is maintained constant $\left(d a^{\prime}=0\right)$. But for an elliptic deformation the reverse is the case.

At first sight it may appear strange that an elliptic deformation should be capable of raising the pitch. But we must remembur that we are here dealing with a vibration such that the phase at both ends of the minor axis is the opposite of that at the centre. A parallel case which admits of complete calculation is that of the rectangle regarded as a deformed square, and vibrating in the gravest symmetrical mode *. It is easily shown that a departure from the square form raises the pitch. Of course, the one-dimensional vibration parallel to the longer side has its pitch depressed.

LX. On some Investigations of the Spectra of Carbon and Hydrocarbon. By Charles W. RafFety, F.R.A.S. $\dagger$

[Plate XIII.]

TWHE spectrum of the Bunsen flame (the "Swan" spectrum) has been made the object of a vast amount of experimental work directed towards the determination of its chemical origin, a résumé of which is given by Dr. Marshall Watts in a paper in the 'Philosophical Magazine' of July $1914 \ddagger$.

The present communication gives the results of a photographic study of the "Swan" spectrum obtained from different sources, and is intended to direct attention to certain features which appear to be worthy of further research-namely, the physical significance of the changes produced in the spectrum by a change in the conditions

* Theory of Sound, $\$ 267(p=q=2)$.

+ Communicated by the Author.

+ "On the Spectra given by Carbon and some of its Compounds; and, in particular, the 'Swan "Spectrum," W. Marshall Watts, Phil. Mag. July 1914. 